\title{
Effects of walnut consumption on cognitive performance in young adults
}

\author{
Peter Pribis $^{1 *}$, Rudolph N. Bailey ${ }^{2}$, Andrew A. Russell ${ }^{2}$, Marcia A. Kilsby ${ }^{3}$, Magaly Hernandez ${ }^{1}$, \\ Winston J. Craig ${ }^{1}$, Tevni Grajales ${ }^{2}$, David J. Shavlik ${ }^{4}$ and Joan Sabatè ${ }^{5}$ \\ ${ }^{1}$ Department of Nutrition and Wellness, Andrews University, 8475 University Boulevard, Marsh Hall 313, Berrien Springs, MI \\ 49104-0210, USA \\ ${ }^{2}$ Department of Educational and Counseling Psychology, Andrews University, 4195 Administration Drive, Bell Hall 159, \\ Berrien Springs, MI 49104, USA \\ ${ }^{3}$ Department of Clinical and Laboratory Sciences, Andrews University, 4270 Administration Drive, Halenz Hall 327, Berrien \\ Springs, MI 49104, USA \\ ${ }^{4}$ Department of Epidemiology and Biostatistics, School of Public Health, Loma Linda University, 11234 Anderson Street, \\ Nichol Hall 2005, Loma Linda, CA 92354, USA \\ ${ }^{5}$ Department of Nutrition, School of Public Health, Loma Linda University, 11234 Anderson Street, Nichol Hall 1102, Loma \\ Linda, CA 92354, USA \\ (Submitted 18 January 2011 - Final revision received 28 June 2011 - Accepted 5 July 2011 - First published online 19 September 2011)
}

\section{Abstract}

Walnuts contain a number of potentially neuroprotective compounds like vitamin E, folate, melatonin, several antioxidative polyphenols and significant amounts of $n-3 \alpha$-linolenic fatty acid. The present study sought to determine the effect of walnuts on verbal and non-verbal reasoning, memory and mood. A total of sixty-four college students were randomly assigned to two treatment sequences in a crossover fashion: walnuts-placebo or placebo-walnuts. Baseline data were collected for non-verbal reasoning, verbal reasoning, memory and mood states. Data were collected again after 8 weeks of intervention. After 6 weeks of washout, the intervention groups followed the diets in reverse order. Data were collected once more at the end of the 8-week intervention period. No significant increases were detected for mood, non-verbal reasoning or memory on the walnut-supplemented diet. However, inferential verbal reasoning increased significantly by $11.2 \%$, indicating a medium effect size $(P=0.009 ; d=0.567)$. In young, healthy, normal adults, walnuts do not appear to improve memory, mood or non-verbal reasoning abilities. However, walnuts may have the ability to increase inferential reasoning.

Key words: Walnuts: Intelligence: Memory: Mood: Inference

In the last 20 years, nutritional neuroscience has emerged as a recognised discipline with the potential to make significant contributions to our understanding of the relationship between nutrition and cognitive functions ${ }^{(1)}$. Some research has been conducted on animals and humans indicating that different neural systems might be affected by dietary manipulations including fruits, vegetables, nuts and fish ${ }^{(2-6)}$.

For the central nervous system to function well, it needs sufficient amounts of water, vitamins such as folate, thiamine, vitamins $\mathrm{B}_{6}$ and $\mathrm{B}_{12}, \alpha$-lipoic acid, lutein and $n-3$ fatty acids $(\mathrm{FA})^{(7,8)}$. Walnuts contain a number of potentially neuroprotective compounds like vitamin $\mathrm{E}$, folate, melatonin, numerous polyphenolics and significant amounts of $n-3$ $\alpha$-linolenic acid (ALA; $18: 3 n-3)^{(9-12)}$. ALA is the precursor for EPA (20:5n-3) and DHA (22:6n-3). DHA is important for neuronal membrane stability, neuroplasticity, synaptic plasticity, gene expression, cell migration and apoptosis. It increases the speed of signal transduction, neurotransmission and increases serotonin and dopamine concentrations. EPA regulates the synthesis of arachidonic acid (20:4n-6) and modulates important inflammatory and immune functions $^{(13-15)}$. The types of FA that are available for the composition of cell membranes and subsequent cognitive processing depend upon diet.

Cognition can be defined narrowly by mental processes like perception, recognition, reasoning, deduction and intuition, and broadly by aggregated concepts like intelligence, memory or affect. Most of the research on the relationship between nutrition and cognitive functions has been done using animals or pathological populations ${ }^{(2-6,16)}$. As far as

Abbreviations: ALA, $\alpha$-linolenic acid; APM, Advanced Progressive Matrices; FA, fatty acid; POMS, Profile of Mood States; WGCTA, Watson-Glaser Critical Thinking Appraisal; WMS-III, Wechsler Memory Scale-Third Edition.

*Corresponding author: Dr P. Pribis, fax +1 269471 3485, email pribis@andrews.edu 
the authors know, the effect of walnut consumption on cognition has never been studied in healthy volunteers. Thus, the present study was designed to investigate the effect of dietary walnut supplementation on cognition and mood in college-age students, using two different intelligence tests - a memory test and a mood test.

\section{Methods}

\section{Subjects}

Following an advertisement campaign on the campus of Andrews University (Berrien Springs, MI, USA), 273 subjects responded with interest to participate in the study. Subjects were disqualified if they were not students, not between the ages of 18 and 25 years, indicated that they would not be present on the campus the whole school year 2009/2010, did not complete a comprehensive lifestyle survey and a short FFQ to assess $n-3$ FA intake ${ }^{(17)}$, had known food allergies or if they did not participate in a comprehensive screening interview (Fig. 1).

\section{Experimental design}

We have used a double-blind, randomised, placebocontrolled, crossover design (Fig. 2) ${ }^{(18)}$. The sixty-four selected subjects were assigned using random allocation software $^{(19)}$ to one of the two study groups - walnuts-placebo and placebo-walnuts - (each of which contained thirty-two subjects). Participants in one experimental group consumed the test meal (banana bread with walnuts) for the first 8 weeks followed by 6 weeks of washout and another 8 weeks of placebo diet (banana bread without walnuts); the other experimental group followed the diets in reverse order. The participants were asked to maintain their usual activities and other lifestyle habits throughout the duration of the study. The study personnel performing measurements and analyses were blinded to the subjects' diet sequence.

This study was conducted according to the guidelines laid down in the Declaration of Helsinki and all procedures involving human subjects were approved by the Institutional Review Board of Andrews University. Written informed consent was obtained from all subjects. Subjects were offered a $\$ 200$ honorarium for their participation.

\section{Dietary intervention}

Participants were asked to consume three slices of banana bread every day for 16 weeks ( 8 weeks banana bread with walnuts and 8 weeks banana bread without walnuts). For the dietary supplementation, we used English walnuts (Juglans regia $\mathrm{L}$.). The two banana breads (walnut and placebo) were baked at the university's kitchen and had very similar appearance and taste. For the banana bread with walnuts, fresh, finely ground walnuts were mixed into the dough and baked at $177^{\circ} \mathrm{C}\left(350^{\circ} \mathrm{F}\right)$ for $1.5 \mathrm{~h}$. Three slices of banana bread with walnuts contained two servings ( $1 / 2$ cup or $60 \mathrm{~g}$ ) of ground walnuts $(6.80 \mathrm{~g}$ of ALA). The daily ration of banana bread without walnuts contained only $0.56 \mathrm{~g}$ of $n-3$ FA coming mainly from margarine.

Once per week, the subjects visited the Nutrition and Wellness Department and received fresh supplies (three loaves twenty-one slices) of banana bread for the upcoming week. Participants were asked to refrigerate the banana bread to prevent spoilage. During the visit, each subject's body weight was measured (without shoes and heavy clothing).

Each week, a sample slice of the banana bread batches (walnut and placebo) was collected and kept frozen until the end of the study. Mixed samples were analysed for levels of macronutrients and FA (Covance Laboratories, Madison, WI, USA). The composition of each of the banana breads is presented in Table 1.

\section{Adherence to dietary intervention}

Adherence was measured by self-report and biological indicators. At the weekly clinics, subjects reported in a form how many slices of the banana bread they consumed daily and at what times during the day. Fasting whole blood was collected at the end of each dietary intervention period. Plasma was separated and stored at $-80^{\circ} \mathrm{C}$ for later analysis. At the end of the study, linoleic acid (LA; $18: 2 n-6)$ and ALA were measured as FA methyl esters through the use of GC (Columbia Analytical Services, Kelso, WA, USA) ${ }^{(20,21)}$.

\section{Cognitive testing}

The cognitive and mood functioning of the students were tested at baseline and at the end of each of the two 8-week treatment periods. One university academic semester is 15 weeks long. Each intervention was designed to run between weeks two and ten of the university semester calendar to avoid the stress associated with the beginning of a new semester or final exams, thus providing a stable environment for the cognitive testing. Students who tested first at the baseline were also tested first and at the same time of the day at the end of each intervention period, to ensure that all subjects had similar testing conditions.

Three different instruments were used to assess memory, non-verbal reasoning and verbal reasoning. Raven's Advanced Progressive Matrices (APM) ${ }^{(22)}$ was administered without time restrictions, and was used to measure general intellectual capacity, and specifically, the ability to solve complex nonverbal problems. Raven's APM is composed of Set I and Set II; Set I was given at the first testing point as a practice test, but only the data from Set II were included in the analysis. The reliability and validity of the test have been established across a range of populations, including African Americans and other non-Whites ${ }^{(23)}$.

The Watson-Glaser Critical Thinking Appraisal (WGCTA) was used to assess students' verbal reasoning faculties, which incorporate the attitude of enquiry, the need for evidence to support conclusions, knowledge of the rules of argument, and skills in integrating these components. The WGCTA is designed to measure different, though interdependent, aspects of critical thinking through its subtests: inference, 


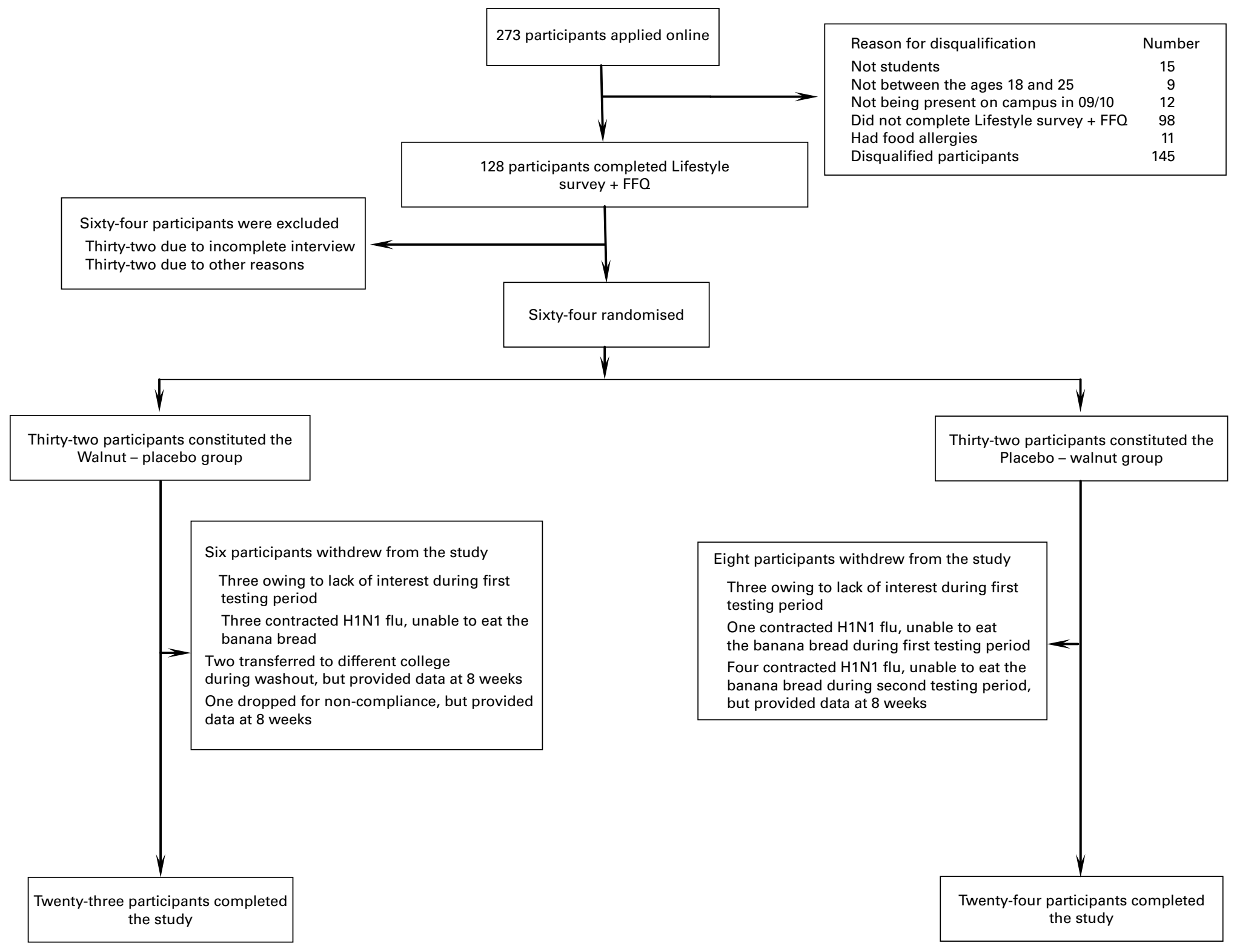

Fig. 1. Participant flow diagram. 


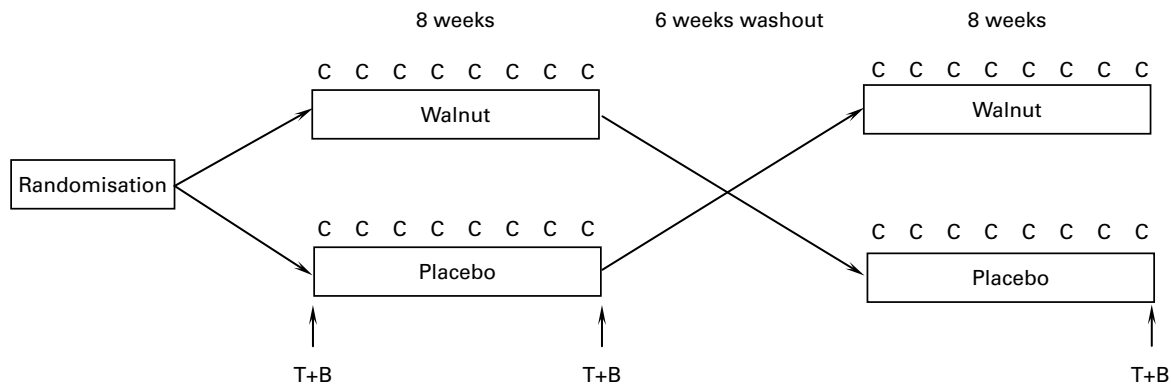

Fig. 2. Experimental design. T, Cognitive testing; B, blood drawing; C, weekly clinic: weight, test meal distribution, compliance assessment.

recognition of assumption, deduction, interpretation, and evaluation of arguments. Content on the WGCTA test reflects both 'neutral' items and 'controversial' items, which relate to social, economic and political subjects. In this way, the WGCTA also reflects critical thinking abilities in the presence of emotional and cognitive biases. The WGCTA is distinguished with a long history of development and use in different countries and different settings ${ }^{(24)}$. The WGCTA was found to have adequate content validity, construct validity, reliability over time and estimates of internal consistency ${ }^{(25)}$.

Wechsler Memory Scale - Third Edition (WMS-III) was used to comprehensively evaluate brain-behaviour relationships involved in learning and memory. WMS-III recognises immediate, delayed and working memory. Each domain is tested in two modalities, auditory and visual, and in two formats, recall and recognition. Data were derived from scores on subtests and these eight indexes: immediate memory, auditory immediate, auditory delayed, auditory recognition delayed, visual immediate, visual delayed, general memory and working memory. All core subtests were administered; optional subtests were excluded from administration. WMS-III has adequate internal-consistency reliability and validity properties ${ }^{(26)}$

The Profile of Mood States (POMS) questionnaire was used to estimate the intensity of mood disturbance in participants. The POMS is one of the most widely used and accepted mood scales in studies of cognition ${ }^{(27,28)}$. The POMS covers six mood domains: tension-anxiety, depression-dejection, anger-hostility, vigour-activity, fatigue-inertia and confusion-bewilderment. The total mood disturbance score is computed by summing the five negative domain scores and subtracting the vigour score. Thus, higher scores indicate a greater degree of mood disturbance.

\section{Statistical analysis}

The sample size determination was based on $5 \%$ significance, $95 \%$ power and measurable medium to large effect size of 0.5-0.7 SD. Allowing for a 30\% dropout rate, the total sample size required for the study was sixty-four individuals.

Primary analysis was carried out as per-protocol analysis. Descriptive values are expressed as means and standard deviations or percentages. All analyses were carried out for both sexes combined. For the crossover analysis, the SAS statistical software (version 9; SAS institute, Cary, NC, USA) used the mixed models procedure (PROC MIXED). Sequence, treatment and period were fixed effects and subject was treated as a random effect. We tested for possible interactions between the dietary treatment and the sequence of the testing period. When differences reached significance, effect sizes (Cohen's $d^{(29)}$ ) were calculated for determination of the clinical relevance of the observed effects. A $d$ between $0 \cdot 20$ and 0.49 was considered to represent a small effect, a $d$ between 0.50 and 0.79 was considered moderate and a $d$ greater than or equal to $0 \cdot 80$ was considered a large effect ${ }^{(30)}$. We used the SPSS (version 18.0; SPSS, Inc., Chicago, IL, USA) to perform independent $t$ tests and $\chi^{2}$ tests for baseline characteristics of participants, and paired $t$ tests to compare the changes in FA levels in plasma and weight change over time. Two-sided $P$ values less than or equal to 0.05 were considered statistically significant.

\section{Results}

The characteristics of the study population are described in Table 2. The participants were between 18 and 25 years old. The mean BMI for males was 23.3 (SD 3.0$) \mathrm{kg} / \mathrm{m}^{2}$, and it was 22.8 (sD 3.6$) \mathrm{kg} / \mathrm{m}^{2}$ for females. The mean $n-3$ FA intake was $0.4(\mathrm{SD} 0.6) \mathrm{g} / \mathrm{d}$ for males and $0.9(\mathrm{SD} 1.1) \mathrm{g} / \mathrm{d}$ for females at baseline. Approximately half the number of participants were Caucasians, followed by African Americans, Hispanics, Asians and people of mixed ethnicity. The majority (90\%) of participants were undergraduate students. The groups had similar exercise habits. The majority (61\%) of students had normal weight and were non-vegetarians (60\%). There were no differences for all of the above characteristics among the study group.

Table 1. Composition of the test meal (banana bread) per $100 \mathrm{~g}^{\star}$

\begin{tabular}{lrr}
\hline & Walnuts & Placebo \\
\hline Energy (kJ/kcal) & $1612 / 385$ & $1202 / 287$ \\
Carbohydrate (g) & 40.9 & 48.9 \\
Protein (g) & $7 \cdot 0$ & $5 \cdot 2$ \\
Fat $(\mathrm{g})$ & 21.5 & $7 \cdot 9$ \\
SFA $(\mathrm{g})$ & $2 \cdot 5$ & 1.5 \\
MUFA (g) & $3 \cdot 8$ & 1.9 \\
PUFA (g) & $14 \cdot 1$ & $4 \cdot 0$ \\
$\alpha$-Linolenic acid $(18: 3 n-3)(\mathrm{g})$ & $2 \cdot 5$ & 0.2 \\
Linoleic acid $(18: 2 n-6)(\mathrm{g})$ & $12 \cdot 2$ & 4.0
\end{tabular}

* Values obtained by chemical analysis (Covance Laboratories, Madison, WI, USA). 
Table 2. Baseline characteristics of the participants

(Mean values, standard deviations, number of participants and percentages, $n$ 64)

\begin{tabular}{|c|c|c|c|c|c|}
\hline \multirow[b]{2}{*}{ Group } & \multicolumn{2}{|c|}{$\begin{array}{c}\text { Walnuts- } \\
\text { placebo }(n \text { 32) }\end{array}$} & \multicolumn{2}{|c|}{$\begin{array}{c}\text { Placebo- } \\
\text { walnuts ( } n \text { 32) }\end{array}$} & \multirow[b]{2}{*}{$P$} \\
\hline & $n$ & $\%$ & $n$ & $\%$ & \\
\hline Age (years) & & & & & $0.872^{*}$ \\
\hline Mean & & & & & \\
\hline SD & & & & & \\
\hline Hours of sleep (h) & & & & & $0.510^{*}$ \\
\hline Mean & & & & & \\
\hline SD & & & & & \\
\hline BMI $\left(\mathrm{kg} / \mathrm{m}^{2}\right)$ & & & & & $0.471^{*}$ \\
\hline Mean & & & & & \\
\hline SD & & & & & \\
\hline$n-3$ intake $(g)$ & & & & & $0.730^{*}$ \\
\hline Mean & & & & & \\
\hline SD & & & & & \\
\hline Ethnicity & & & & & $0.255 \dagger$ \\
\hline Caucasian & 18 & $56 \cdot 3$ & 11 & 35.5 & \\
\hline African American & 5 & $15 \cdot 6$ & 7 & $22 \cdot 6$ & \\
\hline Other & 9 & $28 \cdot 1$ & 13 & 41.9 & \\
\hline Marital status & & & & & $0.162 \dagger$ \\
\hline Single & 31 & $96 \cdot 9$ & 28 & 87.5 & \\
\hline Class standing & & & & & $0.977 \dagger$ \\
\hline Freshman & 8 & $25 \cdot 0$ & 7 & $22 \cdot 6$ & \\
\hline Sophomore & 7 & 21.9 & 9 & $29 \cdot 0$ & \\
\hline Junior & 8 & $25 \cdot 0$ & 7 & $22 \cdot 6$ & \\
\hline Senior & 6 & $18 \cdot 8$ & 5 & $16 \cdot 1$ & \\
\hline Graduate & 3 & 9.4 & 3 & $9 \cdot 7$ & \\
\hline Exercise (30 min/d per week) & & & & & $0.722 \dagger$ \\
\hline $0-2 d$ & 15 & $46 \cdot 9$ & 12 & 38.7 & \\
\hline $3-4 d$ & 12 & 37.5 & 12 & 38.7 & \\
\hline $5-6 d$ & 5 & $15 \cdot 6$ & 7 & $22 \cdot 6$ & \\
\hline Weight category & & & & & $0.459 \dagger$ \\
\hline Underweight (BMI < $18.4 \mathrm{~kg} / \mathrm{m}^{2}$ ) & 3 & $9 \cdot 4$ & 4 & $13 \cdot 3$ & \\
\hline Normal weight (BMI $18.5-24.9 \mathrm{~kg} / \mathrm{m}^{2}$ ) & 22 & $68 \cdot 8$ & 16 & 53.3 & \\
\hline Overweight/obese $\left(\mathrm{BMI}>25.0 \mathrm{~kg} / \mathrm{m}^{2}\right)$ & 7 & $21 \cdot 9$ & 10 & 33.3 & \\
\hline Vegetarian status & & & & & $0.595 \dagger$ \\
\hline Lacto-ovo-vegetarian/vegan & 12 & 37.5 & 13 & $40 \cdot 6$ & \\
\hline Pesco-vegetarian & 1 & $3 \cdot 1$ & & & \\
\hline Omnivore & 19 & 59.4 & 19 & $59 \cdot 4$ & \\
\hline
\end{tabular}

* Independent means $t$ test.

$\dagger \chi^{2}$ test.

Over the course of the study, seventeen (27\%) subjects dropped out. Six withdrew during the first week of the study because of lack of interest, two transferred to a different college between the fall and spring semesters, eight were excluded because they contracted the H1N1 flu and were not able to consume the test meal for several weeks, and one was dropped from the study because of non-compliance. The dropout rate was very similar in both experimental groups. According to the compliance sheets, participants consumed $98 \%$ of the test meals. Mean plasma concentrations of ALA and LA increased 68 and $11 \%$, respectively, while participants were consuming the walnut test meal compared to placebo (Table 3).

The majority of the students (52\%) consumed one slice of banana bread at a time, $26 \%$ consumed two slices at a time and $22 \%$ consumed all the three slices on one occasion. Most students consumed the banana bread for breakfast (42\%), while the rest distributed it equally either for lunch, dinner or snacks. Consumption of three slices of banana bread daily modified the food intake of the participants. Three slices of banana bread with walnuts represented $4354 \mathrm{~kJ}$ ( $1040 \mathrm{kcal})$, and three slices of banana bread without walnuts $3245 \mathrm{~kJ}$ ( $775 \mathrm{kcal})$. Using an FFQ, we monitored the food displacement and observed that during the dietary intervention, males consumed less milk, cereals, pasta and bread but drank more soya or rice milk; females consumed less

Table 3. Plasma concentrations $(\mu \mathrm{g} / \mathrm{ml})$ of linoleic and $\alpha$-linolenic acids at the end of each dietary intervention

(Mean values and standard deviations)

\begin{tabular}{lrrrrrr}
\hline & \multicolumn{2}{c}{$\begin{array}{c}\text { Walnuts } \\
(n \text { 47) }\end{array}$} & & & \multicolumn{2}{c}{$\begin{array}{c}\text { Placebo } \\
(n \text { 47) }\end{array}$} \\
\cline { 2 - 3 } Fatty acid (common name) & Mean & SD & & Mean & SD & $P$ \\
\hline $18: 2 n-6$ (linoleic acid) & 248.3 & 88.3 & & 223.6 & 66.4 & $0.013^{*}$ \\
$18: 3 n-3$ ( $\alpha$-linolenic acid) & 9.9 & 6.7 & & 5.9 & 2.3 & $<0.001^{*}$ \\
\hline
\end{tabular}

* Paired $t$ test. 
milk, cheese, dried fruits and cereals. During the first intervention period, the average weight increase for the walnut group was $1.3(\mathrm{SD} 1.3) \mathrm{kg}$ and it was 0.9 (SD 1.0 ) $\mathrm{kg}$ for the placebo group ( $P<0.001$ for both diets). During the second intervention period, weight increased 0.4 (SD 1.4$) \mathrm{kg}$ for the walnut group and 0.5 (SD 2.2) kg for the placebo group ( $P=0.195$ for walnuts; $P=0.279$ for placebo).

Mean scores and the treatment effects for both sexes for the APM and the WGCTA are presented in Table 4. For the APM, all calculations are based on raw scores. No significant changes in the APM were observed. All calculations for the WGCTA scores are based on raw scores. The raw score is the total number of correct responses. We observed a significant, moderate effect size increase in inference, a subtest of the WGCTA $(11.2 \% ; P=0.009 ; d=0.567)$ on walnut diet.

Table 5 presents the mean scores and treatment effects for the WMS-III memory test. All the calculations for the WMSIII are based on scaled scores adjusted for age. No significant changes in any of the subtests were observed. Mean scores and the treatment effects for the POMS are presented in Table 6. All the calculations for the POMS scores are based on raw scores. No significant changes were observed for any of the subsets or the total score.

\section{Discussion}

This randomised, crossover trial showed no effect of walnut supplementation for 8 weeks in college-age healthy males and females on memory, non-verbal reasoning and mood. However, a selective effect was observed for verbal reasoning.

To our knowledge, the present study has been the first to examine the effect of nutritional supplementation on nonverbal reasoning and verbal reasoning in healthy adults. As no effect was observed for the APM, the authors conclude that non-verbal reasoning seems to be stable enough to not be altered by this nutritional intervention. This may be partly explained by the fact that the study population, college students, may be already having high cognitive abilities and so further improvement would be difficult to achieve. Using the 18-25 year olds US norms, mean scores represent the 81 st and 64th percentiles, yielding intelligence quotient equivalent of 113 and 105 for males and females, respectively ${ }^{(22)}$.

The ability to think critically is generally recognised as an important educational objective and is considered a desirable outcome. Inference is the ability to designate the degree of accuracy of conclusions that have been drawn from certain facts. Inferential reasoning is tested in such a way that subjects are read a short narrative followed by five statements. Subjects must make a decision whether the statements are true, partially true, false, partially false or whether there is not enough information to make a judgement. The results for inference showed a significant improvement after walnut supplementation $(P=0.009 ; d=0.567 ; 11.2 \%)$, but we did not observe any period-specific effect. The observed improvement occurred already after 8 weeks of walnut supplementation; nevertheless, whether such effect might persist over a longer period like years or at different life stages, we do not

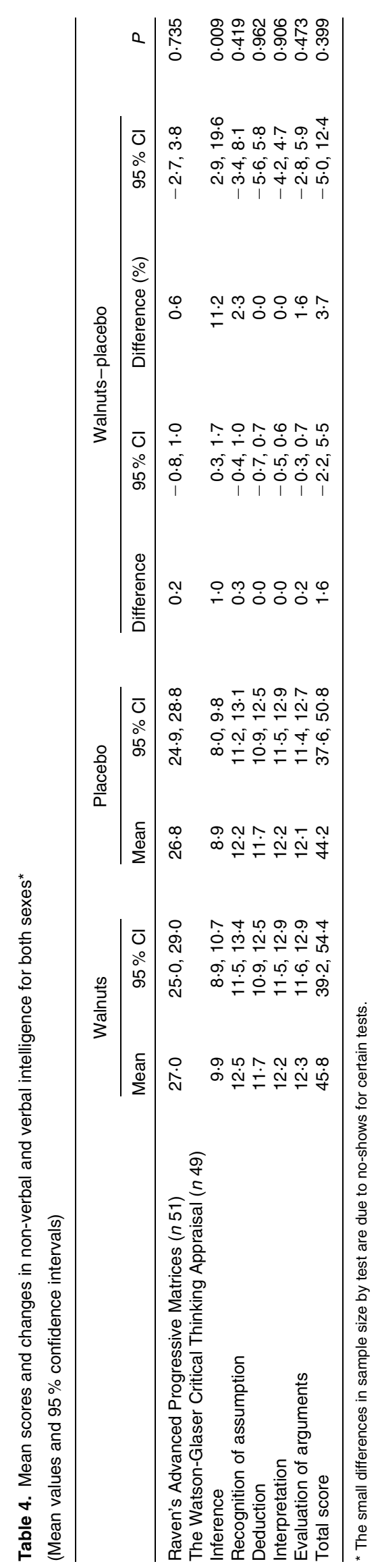


Table 5. Changes in memory for both sexes

(Mean values and $95 \%$ confidence intervals)

\begin{tabular}{|c|c|c|c|c|c|c|c|c|c|}
\hline & \multicolumn{2}{|c|}{ Walnuts } & \multicolumn{2}{|c|}{ Placebo } & \multicolumn{4}{|c|}{ Walnuts-placebo } & \multirow[b]{2}{*}{$P$} \\
\hline & Mean & $95 \% \mathrm{Cl}$ & Mean & $95 \% \mathrm{Cl}$ & Difference & $95 \% \mathrm{Cl}$ & Difference (\%) & $95 \% \mathrm{Cl}$ & \\
\hline \multicolumn{10}{|c|}{ The Wechsler Memory Scale-III ( $n$ 52) } \\
\hline Logical memory I & $12 \cdot 7$ & $11 \cdot 9,13 \cdot 5$ & $12 \cdot 5$ & $11 \cdot 7,13 \cdot 3$ & 0.2 & $-0.5,0.9$ & 1.7 & $-3 \cdot 7,7 \cdot 1$ & 0.525 \\
\hline Faces I & $13 \cdot 1$ & $12 \cdot 2,14 \cdot 0$ & $13 \cdot 1$ & $12 \cdot 2,14 \cdot 0$ & 0.0 & $-0.9,0.9$ & 0.0 & $-6 \cdot 7,6 \cdot 5$ & 0.976 \\
\hline Verbal paired associates I & 14.4 & $13 \cdot 8,15 \cdot 0$ & $14 \cdot 1$ & $13 \cdot 5,14 \cdot 7$ & 0.3 & $-0.2,0.9$ & $2 \cdot 3$ & $-1 \cdot 7,6 \cdot 4$ & 0.255 \\
\hline Family pictures I & $13 \cdot 3$ & $12 \cdot 7,14 \cdot 0$ & $13 \cdot 5$ & $12 \cdot 9,14 \cdot 1$ & $-0 \cdot 2$ & $-0 \cdot 7,0.4$ & $-1 \cdot 2$ & $-5 \cdot 3,2 \cdot 9$ & 0.553 \\
\hline Letter-number sequencing & 11.0 & $10 \cdot 2,11 \cdot 8$ & 11.0 & $10 \cdot 2,11 \cdot 8$ & 0.0 & $-0.6,0.7$ & 0.0 & $-5.5,6.5$ & 0.867 \\
\hline Spatial span & $11 \cdot 3$ & $10 \cdot 4,12 \cdot 1$ & $11 \cdot 7$ & $10 \cdot 9,12 \cdot 6$ & -0.4 & $-1 \cdot 0,0.2$ & $-3 \cdot 8$ & $-8 \cdot 9,1 \cdot 4$ & 0.148 \\
\hline Logical memory II & $13 \cdot 5$ & $12 \cdot 7,14 \cdot 2$ & $13 \cdot 7$ & $13 \cdot 0,14 \cdot 4$ & -0.2 & $-0.7,0.3$ & -1.6 & $-5 \cdot 3,2 \cdot 2$ & 0.405 \\
\hline Faces II & 12.5 & $11.5,13.5$ & 12.5 & $11 \cdot 5,13.5$ & 0.0 & $-0.8,0.9$ & 0.0 & $-6 \cdot 7,7 \cdot 6$ & 0.898 \\
\hline Verbal paired associates II & 11.9 & $11 \cdot 8,12 \cdot 1$ & $12 \cdot 0$ & $11 \cdot 8,12 \cdot 1$ & -0.1 & $-0.2,0.2$ & -0.3 & $-1.9,1.4$ & 0.744 \\
\hline Family pictures II & $13 \cdot 4$ & $12 \cdot 7,14 \cdot 1$ & $13 \cdot 7$ & $13 \cdot 1,14 \cdot 4$ & -0.3 & $-0.9,0.2$ & -2.5 & $-6 \cdot 6,1 \cdot 7$ & 0.238 \\
\hline Auditory recognition & $12 \cdot 6$ & $11.9,13.4$ & $12 \cdot 3$ & $11 \cdot 5,13 \cdot 0$ & 0.3 & $-0.3,1.0$ & $2 \cdot 9$ & $-2 \cdot 0,7 \cdot 8$ & 0.245 \\
\hline Auditory immediate & $27 \cdot 1$ & $26 \cdot 0,28 \cdot 2$ & $26 \cdot 6$ & $25 \cdot 5,27 \cdot 6$ & 0.5 & $-0.3,1.4$ & $2 \cdot 0$ & $-1 \cdot 2,5 \cdot 2$ & 0.221 \\
\hline Visual immediate & $26 \cdot 4$ & $25 \cdot 2,27 \cdot 6$ & $26 \cdot 6$ & $25 \cdot 4,27 \cdot 8$ & -0.2 & $-1 \cdot 2,0.8$ & -0.7 & $-4 \cdot 3,3 \cdot 0$ & 0.703 \\
\hline Immediate memory & 53.5 & $51 \cdot 5,55 \cdot 5$ & $52 \cdot 8$ & $50 \cdot 8,54 \cdot 8$ & 0.7 & $-0 \cdot 7,2 \cdot 2$ & 1.4 & $-1 \cdot 3,4 \cdot 1$ & 0.308 \\
\hline Auditory delayed & $25 \cdot 4$ & $24 \cdot 7,26 \cdot 2$ & $25 \cdot 7$ & $24 \cdot 9,26 \cdot 4$ & -0.2 & $-0.8,0.3$ & $-1 \cdot 0$ & $-3 \cdot 2,1 \cdot 3$ & 0.396 \\
\hline Visual delayed & $25 \cdot 9$ & $24 \cdot 5,27 \cdot 3$ & $26 \cdot 2$ & $24 \cdot 8,27 \cdot 6$ & -0.3 & $-1.4,0.9$ & $-1 \cdot 0$ & $-5 \cdot 4,3 \cdot 3$ & 0.634 \\
\hline Auditory recognition delayed & $12 \cdot 6$ & $11 \cdot 9,13 \cdot 4$ & $12 \cdot 3$ & $11 \cdot 5,13 \cdot 0$ & 0.4 & $-0.3,1.0$ & 2.9 & $-2 \cdot 0,7 \cdot 8$ & 0.245 \\
\hline General memory & $62 \cdot 9$ & $60 \cdot 1,65 \cdot 6$ & 64.2 & $61 \cdot 5,67 \cdot 0$ & $-1 \cdot 3$ & $-3 \cdot 9,1 \cdot 2$ & $-2 \cdot 1$ & $-6 \cdot 1,1 \cdot 8$ & 0.288 \\
\hline Working memory & $22 \cdot 6$ & $21 \cdot 3,24 \cdot 0$ & $22 \cdot 8$ & $21 \cdot 5,24 \cdot 2$ & -0.2 & $-1 \cdot 2,0.8$ & -0.9 & $-5 \cdot 2,3 \cdot 3$ & 0.664 \\
\hline
\end{tabular}

know. The authors are unable to explain why inference alone was affected by consumption of walnuts and not the other 'critical thinking' subtests - recognition of assumption, deduction, interpretation, and evaluation of arguments. All the other subtests either did not change or there was only a very small increase; none of the subtest scores decreased. The WGCTA has a strong internal consistency. Individual subtests correlate highly $(r 0.5-0.8)$ with the total score. In our study, there was a significant direct correlation between inference and the total score $(r 0.8 ; P<0 \cdot 001)$. This would support the idea that single factor results are consistent with the overall data. $n-3$ FA can modulate many of the signal transduction mechanisms operating at the synaptic level, influence pathways with different neurotransmitters such as serotonin, dopamine or acetylcholine, and possibly be responsible for some of the reported effects $^{(31,32)}$. On a practical level, maybe students or young professionals in social studies, mathematics, sciences or other fields that involve a great deal of critical thinking or decision-making could possibly benefit and gain a slight advantage through regular consumption of walnuts. Considering these findings, it is important that further research be conducted in order to gain a deeper understanding of this differential impact of walnuts consumption on this component of verbal reasoning.

Previous studies have been conducted around the effects of $n$-3 FA on mood states with varying results. Most studies appear to focus on depressed populations, and some have found reduced levels of $n-3$ FA in plasma and cell membranes of depressed patients ${ }^{(33-35)}$. Appleton et al. ${ }^{(36)}$ evaluated randomised controlled trials in various populations including healthy samples and concluded, that trial evidence of the effects of $n$-3 FA on depressed mood has increased but remains difficult to summarise. The evidence available provides some support of a benefit of $n-3$ FA in individuals with diagnosed depressive illness but no evidence of any benefit in individuals without a diagnosis of depressive illness. The present study was built around the premise that walnuts are an available, excellent source of $n-3$ FA. As participants in the present study scored around the 50th percentile at baseline (not depressed) ${ }^{(27)}$, the results in our study confirm the present consensus that $n-3$ FA supplementation in nondepressed healthy adults has no effect on mood.

Table 6. Changes in mood for both sexes

(Mean values and $95 \%$ confidence intervals)

\begin{tabular}{|c|c|c|c|c|c|c|c|c|c|}
\hline & \multicolumn{2}{|c|}{ Walnuts } & \multicolumn{2}{|c|}{ Placebo } & \multicolumn{4}{|c|}{ Walnuts-placebo } & \multirow[b]{2}{*}{$P$} \\
\hline & Mean & $95 \% \mathrm{Cl}$ & Mean & $95 \% \mathrm{Cl}$ & Difference & $95 \% \mathrm{Cl}$ & Difference $(\%)$ & $95 \% \mathrm{Cl}$ & \\
\hline \multicolumn{10}{|l|}{ Profiles of moods ( $n$ 49) } \\
\hline Tension-anxiety & $11 \cdot 2$ & $9 \cdot 3,13 \cdot 2$ & 11.9 & $9 \cdot 9,13 \cdot 8$ & -0.7 & $-2 \cdot 5,1 \cdot 2$ & $-5 \cdot 2$ & $-20 \cdot 8,10 \cdot 4$ & 0.507 \\
\hline Depression-dejection & 9.7 & $6 \cdot 7,12 \cdot 7$ & $12 \cdot 1$ & $9 \cdot 1,15 \cdot 2$ & -2.4 & $-5.4,0.5$ & -20.0 & $-44 \cdot 2,4 \cdot 2$ & 0.103 \\
\hline Anger-hostility & $6 \cdot 5$ & $4 \cdot 7,8 \cdot 3$ & 7.5 & $5 \cdot 7,9 \cdot 3$ & $-1 \cdot 0$ & $-2 \cdot 8,0 \cdot 8$ & $-13 \cdot 3$ & $-36 \cdot 8,10 \cdot 2$ & 0.261 \\
\hline Vigour-activity & $13 \cdot 8$ & $12 \cdot 2,15 \cdot 4$ & 13.1 & $11 \cdot 5,14 \cdot 7$ & 0.7 & $-1 \cdot 0,2 \cdot 4$ & $5 \cdot 3$ & $-7.5,18 \cdot 0$ & 0.408 \\
\hline Fatigue-inertia & $10 \cdot 2$ & $8 \cdot 7,11 \cdot 7$ & $10 \cdot 1$ & $8 \cdot 6,11.6$ & $0 \cdot 1$ & $-1 \cdot 4,1 \cdot 7$ & $1 \cdot 1$ & $-14 \cdot 3,16 \cdot 6$ & 0.882 \\
\hline Confusion-bewilderment & 9.5 & $8 \cdot 2,10 \cdot 8$ & 9.5 & $8 \cdot 2,10 \cdot 8$ & 0.0 & $-1 \cdot 2,1 \cdot 2$ & 0.0 & $-12 \cdot 6,12 \cdot 1$ & 0.969 \\
\hline Total score & 33.5 & $24 \cdot 7,42 \cdot 3$ & 37.9 & $29 \cdot 1,46 \cdot 6$ & -4.4 & $-12 \cdot 3,3 \cdot 5$ & -11.6 & $-32.4,9.2$ & 0.267 \\
\hline
\end{tabular}


There is an unfortunate dearth of research on nuts and cognition. One study by Willis et al. ${ }^{(4)}$ reported on the effects of walnuts on cognitive and motor functions in aged rats. Results for the motor testing showed that a $2 \%$ walnut diet improved performance on rod walking, while the $6 \%$ walnut diet improved performance on the medium plank walk; the highest dose of the $9 \%$ walnut diet did not improve psychomotor performance. All of the walnut diets improved working memory in the Morris water maze; nevertheless the 9\% diet showed impaired reference memory. Nooyens et al. ${ }^{(16)}$ recently reported that in the Doetichen Cohort Study, higher intake of nuts was associated with significantly elevated cognitive function (memory, speed, cognitive flexibility and global cognitive function) at baseline. The difference in cognitive functions between the lowest and the highest quintile of nut consumption was equivalent to 5-8 years' difference in age. However, there was no significant change observed in cognitive function at the second testing after 5 years. Both of these studies were conducted on aged or ageing populations, suggesting that the many neuroprotective compounds in nuts possibly slow the cognitive declines that accompany ageing. In our randomised trial, the results of the WMS-III test showed no effects on memory after 8 weeks' walnuts supplementation. Initial cognitive functioning of our population may have been too high for nutrients supplementation to be effective. Using norms, the average score of the different WMS-III subtest at baseline for both sexes ranged between the 50 th and 80 th percentiles ${ }^{(26)}$. These results appear to indicate that the effects of walnuts on healthy adults during a period of short, intensive supplementation are limited.

A limitation of the present study is that the duration of the intervention may have been too short. Another consideration is that the relationship between walnut supplementation and cognitive performance increases may be curvilinear rather than linear. In such a case, future studies may benefit from including experimental groups with varying levels of walnut supplementation in order to identify the optimal amount of walnuts necessary to enhance performance. This is difficult to predict a priori, however, since the mechanisms by which walnuts influence cognitive performance in both the short term and the long term are not completely understood. An additional constraint that we faced in this study was the dropout rate, which was elevated because of the flu epidemic. This attrition rate reduced the level of statistical power available for our analysis. However, the bias that would result from the withdrawal of participants with excellent or poor cognition would probably be negligible.

In conclusion, in this randomised walnuts feeding trial, two different intelligence tests - a memory test and a mood test were used to assess the potential cognition changes in young adults. Results seem to indicate that walnuts increase inferential verbal reasoning. Young, healthy adults involved in fields that require inferential verbal reasoning faculties could possibly experience a modest improvement after regular walnut consumption. Mood, memory and non-verbal reasoning do not appear to be affected by walnuts. Further studies are necessary to corroborate these findings.

\section{Acknowledgements}

The present study was supported in part by the California Walnut Commission (Folton, CA, USA). The funders had no role in the implementation, data collection, management, analysis or interpretation of the study, or in the preparation, review and approval of the manuscript. We are indebted to the participants for their enthusiastic commitment to the study protocol and to Benneth Chilson, Carol Burtnack, Jasmina Popovska, Megan McLoughlin, Galiya Elias, Kristy Koeppe, Allison Ireland, Matt Bonynge, Elizabeth Lundy, Ellen Klinedinst, Jamie Ely, Nathanael Stephens and Lilian M. Correa for their technical assistance. The authors' responsibilities were as follows: P. P. was the principal investigator, who also designed and planned the study, applied for funding and drafted the manuscript. R. N. B. and A. A. R. oversaw the psychological part of the study and helped in the drafting of the manuscript. M. A. K. oversaw the clinical part of the study. M. H. and W. J. C. oversaw the nutritional part of the study. D. J. S. and T. G. were responsible for statistical analysis. J. S. helped to design the study and drafted the manuscript. The corresponding author (P. P.) had full access to all data and had the final responsibility for the decision to submit the manuscript for publication. J. S. is an unpaid emeritus member of the California Walnut Commission Scientific Advisory Board. The authors declare no conflicts of interest pertaining to this study.

\section{References}

1. Wainwright PE (2000) Nutrition and behavior: the role of $n-3$ fatty acids in cognitive functions. Br J Nutr 83, 337-339.

2. Kang JH, Asherio A \& Grodstein F (2005) Fruit and vegetable consumption and cognitive decline in aging women. Ann Neurol 57, 713-720.

3. Morris MC, Evans DA, Tangney CC, et al. (2006) Associations of vegetable and fruit consumption with age-related cognitive change. Neurology 67, 1370-1376.

4. Willis LM, Shukitt-Hale B, Cheng V, et al. (2009) Dosedepended effect of walnuts on motor and cognitive function in aged rats. Br J Nutr 101, 1140-1144.

5. Morley JE \& Banks WA (2010) Lipids and cognition. J Alzheimers Dis 20, 737-747.

6. van Gelder BM, Tijhuis M, Kalmijn S, et al. (2007) Fish consumption, $n-3$ fatty acids, and subsequent 5-y cognitive decline in elderly men: the Zutphen Study. Am J Clin Nutr 85, 1142-1147

7. Grandjean AC \& Grandjean NR (2007) Dehydration and cognitive performance. J Am Coll Nutr 26, 549S-554S.

8. Morley JE (2010) Nutrition and the brain. Clin Geriatr Med 26, 89-98.

9. Crews C, Hough P, Godward J, et al. (2005) Study of main constituents of some authentic walnut oils. J Agric Food Chem 53, 4853-4860.

10. Fukuda T, Ito H \& Yoshida T (2003) Antioxidative polyphenols from walnuts (Juglans regia L.). Phytochemistry 63, 795-801.

11. Reiter RJ, Manchester LC \& Tan DX (2005) Melatonin in walnuts: influence on levels of melatonin and total antioxidant capacity of blood. Nutrition 21, 920-924.

12. Halvorsen BL, Carlsen MH, Phillips KM, et al. (2006) Content of redox-active compounds (ie, antioxidants) in foods consumed in the United States. Am J Clin Nutr 84, 95-135. 
13. Heinrichs SC (2010) Dietary $\omega$-3 fatty acid supplementation for optimizing neuronal structure and function. Mol Nutr Food Res 54, 447-456.

14. Innis SM (2007) Dietary ( $n$-3) fatty acids and brain development. J Nutr 137, 855-859.

15. Su K-P (2009) Biological mechanism of antidepressant effect of omega-3 fatty acids: how does fish oil act as a "Mind-Body interface"? Neurosignals 17, 144-152.

16. Nooyens ACJ, Bueno-de-Mesquita HB, van Boxtel MPJ, et al. (2011) Fruit and vegetable intake and cognitive decline in middle-aged men and women: the Doetinchem Cohort Study. Br J Nutr 106, 752-761.

17. Ritter-Gooder PK, Lewis NM, Heidal KB, et al. (2006) Validity and reliability of a quantitative food frequency questionnaire measuring $n-3$ fatty acid intake in cardiac patients in the Midwest: a validation pilot study. J Am Diet Assoc 106, $1251-1255$.

18. Evans AN \& Rooney BJ (2011) Methods in Psychological Research, 2nd ed. Thousand Oaks, CA: SAGE Publications, Inc.

19. Saghaei M (2004) Random allocation software for parallel group randomized trials. BMC Med Res Methodol 4, 26.

20. Zock PL, Mensik RP, Harryvan J, et al. (1997) Fatty acids in serum cholesteryl esters as quantitative biomarkers of dietary intake in humans. Am J Epidemiol 145, 1114-1122.

21. Lepage G \& Roy CC (1986) Direct transesterification of all classes of lipids in a one-step-reaction. J Lipid Res 27, $114-120$.

22. Raven J, Raven JC \& Court JH (1998) Manual for Raven's Advanced Progressive Matrices, 1998 ed. San Antonio, TX: Harcourt Assessment, Inc.

23. Raven J (2000) The Raven's progressive matrices: change and stability over culture and time. Cognitive Psychol 41, 1-48.

24. El Hassan K \& Madhum G (2007) Validating the Watson Glaser Critical Thinking Appraisal. High Educ 54, 361-383.
25. Watson G \& Glaser EM (1980) Watson-Glaser Critical Think ing Appraisal Manual. San Antonio, TX: The Psychological Corporation - Harcourt Brace \& Co.

26. Wechsler D (2002) Wechsler Adult Intelligence Scale III Third Edition. Technical Manual. San Antonio, TX: The Psychological Corporation - Harcourt Assessment Co.

27. McNair DM \& Heuchert JWP (2005) Profiles of Mood States Technical Update. Toronto, ON: Multi-Health Systems, Inc.

28. Nyenhuis DL, Yamamoto C, Luchetta T, et al. (1999) Adult and geriatric normative data and validation of the profile of mood states. J Clin Psychol 55, 79-86.

29. Cohen J (1998) Statistical Power for the Behavioral Sciences, 2nd ed. Hillsdale, NJ: Lawrence Erlbaum.

30. Warner RM (2008) Applied Statistics. Thousand Oaks, CA: Sage Publications, Inc.

31. Fontani G, Corradeshi F, Felici A, et al. (2005) Cognitive and physiological effects of omega-3 polyunsaturated fatty acids supplementation in healthy subjects. Eur J Clin Invest 35, 691-699.

32. Haag M (2003) Essential fatty acids and the brain. Can J Psychiatry 48, 195-203.

33. Edwards R, Peet M, Shay J, et al. (1998) Omega-3 polyunsaturated fatty acid levels in the diet and in red blood cell membranes of depressed patients. J Affect Disord 48, 149-155.

34. Maes M, Christophe A, Delanghe J, et al. (1999) Lowered omega-3 polyunsaturated fatty acids in serum phospholipids and cholesteryl esters of depressed patients. Psychiatry Res 85, 275-319.

35. Peet M, Murphy B, Shay J, et al. (1998) Depletion of omega-3 fatty acid levels in red blood cell membranes of depressive patients. Biol Psychiatry 43, 315-319.

36. Appleton KM, Rogers PJ \& Ness AR (2010) Updated systematic review and meta-analysis of effects of $n$-3 long-chain polyunsaturated fatty acids on depressed mood. Am J Clin Nutr 91, 757-770. 